\title{
Time-resolved reflectivity technique: improvement and applications
}

\author{
K. M. A. EL-Kader \\ Physics department, Faculty of science, Suez Canal University, Ismailia, Egypt
}

\begin{abstract}
A new method for determination of the reflectivity of Si in different phase transitions during pulsed laser irradiation is presented in this paper. This method is applied on TRR spectra of crystalline silicon (c-Si) in a medium of oxygen and amorphous hydrogenated silicon (a-Si:H). Time resolved reflectivity (TRR) measurements on silicon has been made during pulsed $\mathrm{XeCl}$ excimer laser irradiation $(308 \mathrm{~nm}, 28 \mathrm{~nm}$ FWHM) in a medium of oxygen. The samples were irradiated in the energy density range $400-1100 \mathrm{~mJ} / \mathrm{cm}^{2}$. The reflectivity was measured with a probe He-Ne laser $(632.8 \mathrm{~nm})$. Depending on the energy density of the excimer pulse, heating, melting and resolidification of the surface were monitored by TRR spectra. From these measurements we were able to determine the melting threshold energy density for c-Si, depending on the energy densities, time of melting and maximum reflectivity have been measured. TRR spectra of a sample with $3 \mu \mathrm{m}$ thick a-Si layer for first shot of measurements were calibrated. A series of a-Si : $\mathrm{H}$ samples of the same thickness $(0.34 \mu \mathrm{m})$ irradiated with a constant energy density $450 \mathrm{~mJ} / \mathrm{cm}^{2}$ and the three consecutive TRR spectra of the irradiated samples were calibrated.
\end{abstract}

\section{INTRODUCTION}

Ultrathin (10-100 Å) silicon oxide layer on silicon are of fundamental interest because of their application in MIS solar cells [1]. Low temperature processing is desirable in order to prevent substrate degradation in the production of semiconductor-oxide overlayers. Many processes have been proposed for Si which include chemical deposition (CVD), laser-induced CVD and laser irradiation in oxygen environment [2].

Among other advantages, laser processing offers the interesting possibility of local oxidation (to micronsized area). When pulsed laser irradiation is used, high temperatures are achieved at the near-surface region during short period of time, which ensures rapid and localized processing. Several works have shown the existence of enhancement of the oxidation process by irradiating with visible and ultraviolet UV light $[3,4]$. TRR measurements had been used to provide information on whatever the process occurs in liquid or solid phase in addition to record the reflectivity changes as oxidation proceeds [2]. To our knowledge the melting threshold, time of melting as a function of energy density and maximum reflectivity depending on energy density are not determined.

Recrystallization of thin amorphous silicon (a-Si) layers using light sources was proved to be a suitable method for preparing polycrystalline silicon (pc-Si) [5] which is used in the electronics industry, e.g., as metal gate in metal-oxide-semiconductor transistors.

Several groups [6-8] have investigated the transformation of amorphous silicon to a polycrystalline phase. Recently TRR measurements have been performed to monitor the appearance and time evolution of various phase transitions in amorphous hydrogenated silicon (a-Si : H) during irradiation [7]. A lot of information are missing because the reflectivity of the surface is unknown and only roughly description for the reflectivity was done.

In this paper, we develop a new method for determination the reflectivity in TRR spectrum during pulsed excimer laser irradiation. It is applied on TRR spectra of c-Si irradiated with light from $\mathrm{XeCl} 308 \mathrm{~nm}$ in environment of oxygen and a-Si : $\mathrm{H}$ spectra.

\section{MATERIALS AND METHODS}

The experimental set-up is similar to those usually used for recent TRR measurements [9]. The c-Si samples were irradiated in oxygen environment in a stainless steel chamber. $\mathrm{An} \mathrm{XeCl}(\lambda=308 \mathrm{~nm})$ excimer laser (LAMBDA LPX $250 \mathrm{iC}$ ) with $28 \mathrm{~ns}$ ffull width at halfmaximum (FWHM)\} pulses of roughly Gaussian shape has been used. The homogeneous part of the beam has been transmitted through a rectangular diaphragm. During the experiment, the energy of the individual pulses was measured with the pyrometer Coherent Labmaster equipped with measuring head LM-5 (the accuracy of the relative value is better than 5\%). The energy density on the sample surface was controlled by focusing the beam with a spherical fused silica lens ( $f=180 \mathrm{~mm}$ ) moved carefully by a micropositioner. Typical dimensions of the laser spot on the sample were approx. $1-2 \times 2-4 \mathrm{~mm}^{2}$ and were determined with an accuracy of better than $5 \%$. A probe beam of HeNe laser $(\lambda=632.8 \mathrm{~nm}, 0.5 \mathrm{mw}$ and unpolarized beam) was focused in a spot with a diameter of approximately $500 \mu \mathrm{m}$ and angle of incidence $30^{\circ}$. The reflected beam was passed through an interference filter and its intensity was measured by Si photodiode (rise time $<1 \mathrm{~ns}$ ). Photodiode signals were amplified with short-range amplifier (10-100 MHZ) and captured by the oscillograph Philips PM 3323. Data were digitalized and processed by a personal computer.

Si samples used in these experiments were cut from $0.35 \mathrm{~mm}$ thick wafer of the Czochralski grown, p-type Si (100)-oriented single crystal (5-12 $\Omega \mathrm{cm})$, polished. It 
was necessary to clean all samples with appropriate solvents to remove any organic surface contaminants just before the experiment. The native oxide layer was removed by irradiating Si surface under vacuum with 120 pulses at energy density $450 \mathrm{~mJ} / \mathrm{cm}^{2}$ [10]. The chamber was evacuated from air and filled with oxygen.

The a-Si : H layers have been prepared by a radio frequency glow discharge deposition [7]. The samples prepared with the following typical parameters: As the substrate, 7059 Corning glass plates covered with two evaporated strip $\mathrm{Cr}$ electrodes, the thickness of the samples was $0.34 \mu \mathrm{m}$ irradiated with a variable number of pulses with a constant energy density of $0.45 \mathrm{~J} / \mathrm{cm}^{2}$ which maximize the photoluminescence intensity. Substrate temperature $250{ }^{\circ} \mathrm{C}$, working pressure $20 \mathrm{~Pa}, \mathrm{SiH}_{4}$ flow rate $30 \mathrm{sccm}$ and deposition rate of approximately $1 \mu \mathrm{m}$ per hour.

\section{RESULTS AND DISCUSSION}

3.1. Determination of the reflectivity in TRR. spectrum This method allows us to determine the reflectivity of any bulk or thin film semiconductors at $632.8 \mathrm{~nm}$ (HeNe laser) during pulsed excimer laser irradiation. TRR spectrum of c-Si at energy density $\geq 900 \mathrm{~mJ} / \mathrm{cm}^{2}$ will taken as a reference for the calibration of any TRR spectrum because there is $38.3 \%$ difference between the reflectivity of solid Si and the reflectivity of liquid Si at the same probe wavelength $632.8 \mathrm{~nm}$. In our experiment, Si photodiode has high sensitivity to low and rapidly changing light levels and good linearity of the detector's electrical response in following both rising and falling light signals.

It was reported that the reflectivity of He-Ne laser from silicon surface during pulsed excimer laser irradiation is influenced by a lot of parameters such as the incident laser pulse shape, medium of irradiation and the Si surface condition for more details see [11]. For this reason Si surface was cleaned from the native oxide layer and Si was irradiated under vacuum.

To determine the reflectivity of any bulk or thin film semiconductor materials in TRR spectrum the following procedures were followed

(1) Clean Si sample was irradiated by pulsed excimer laser under vacuum with 30 pulses at energy density range 900 to $1080 \mathrm{~mJ} / \mathrm{cm}^{2}$. The obtained TRR spectra were analysized and it was found that the average reflected intensity is equal $0.135 \mathrm{mV}$. Figuer 1 represents an example of TRR spectrum on clean c-Si (100) surface irradiated by $\mathrm{XeCl}$ excimer laser at $E=1077 \mathrm{~mJ} / \mathrm{cm}^{2}$. In Figure 1 the reflected intensity of the photodiode $(0.135 \mathrm{mV})$ is equivalent to $38.3 \%$. This value represent difference between reflectivity of liquid silicon $R_{\mathrm{l}}=73 \%$ and the reflectivity of solid $\mathrm{Si}$ at room temperature $\left(R_{\mathrm{C}}=34.7 \%\right)$.

(2) Multiply reflectivity column (mV) in TRR spectrum by the factor $F$

$$
F=\frac{R_{\mathrm{l}}(\%)-R_{\mathrm{S}}(\%)}{R_{\mathrm{l}}(\mathrm{mV})}=284
$$

where $R_{l}(\%)$ : reflectivity of liquid $\mathrm{Si}$ at $632.8 \mathrm{~nm}=73 \%$ [12] $R_{\mathrm{S}}$ (\%): reflectivity of solid $\mathrm{Si}$ at $632.8 \mathrm{~nm}$ and room temperature $=34.7 \%[13] . R_{\mathrm{l}}(\mathrm{mV})$ : The maximum reflectivited intensity of clean $\mathrm{Si}$ at $E=1077 \mathrm{~mJ} / \mathrm{cm}^{2}$ from Figure 1 it is equal to $0.135 \mathrm{mV}$.

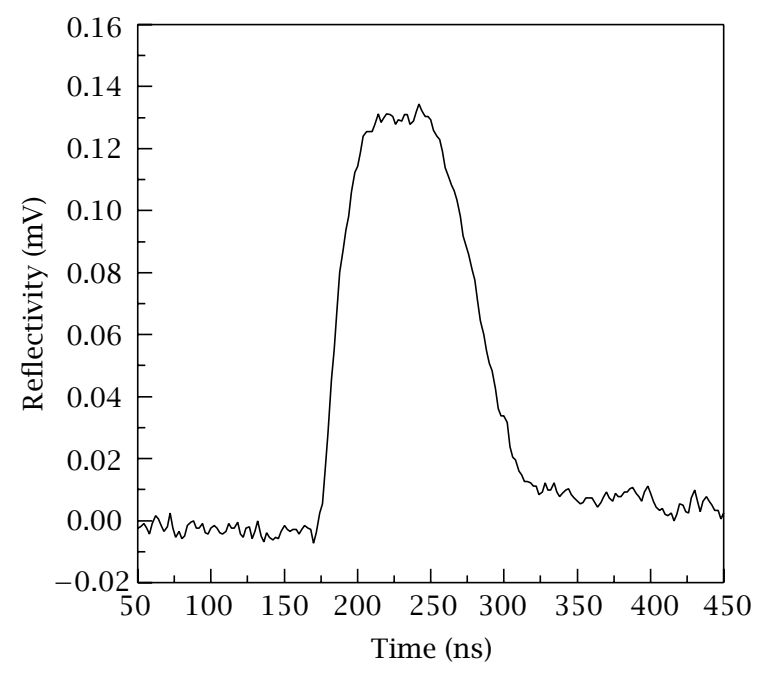

Figure 1. Time-resolved reflectivity spectrum for Probe HeNe laser at $E=1077 \mathrm{~mJ} / \mathrm{cm}^{2}$.

(3) Add the value of the reflectivity of the solid material (a-Si, $\mathrm{SiO}_{2}$, etc.) at room temperature and at $632.8 \mathrm{~nm}$ to the reflectivity column.

3.2. Application of the reflectivity method on Si irradiated in oxygen environment using pulsed excimer laser. c-Si sample covered with native oxide layer was irradiated in stainless steel chamber filled with oxygen in the energy density range from 400 to $1100 \mathrm{~mJ} / \mathrm{cm}^{2}$. TRR spectra which have been obtained during irradiation of c-Si were calibrated as in the previous section where $F=284$ and $R_{\mathrm{S}}=35 \%$ [14]. Plotting maximum reflectivities of TRR spectra as a function of energy densities, the experimental points in Figure 2. were obtained.

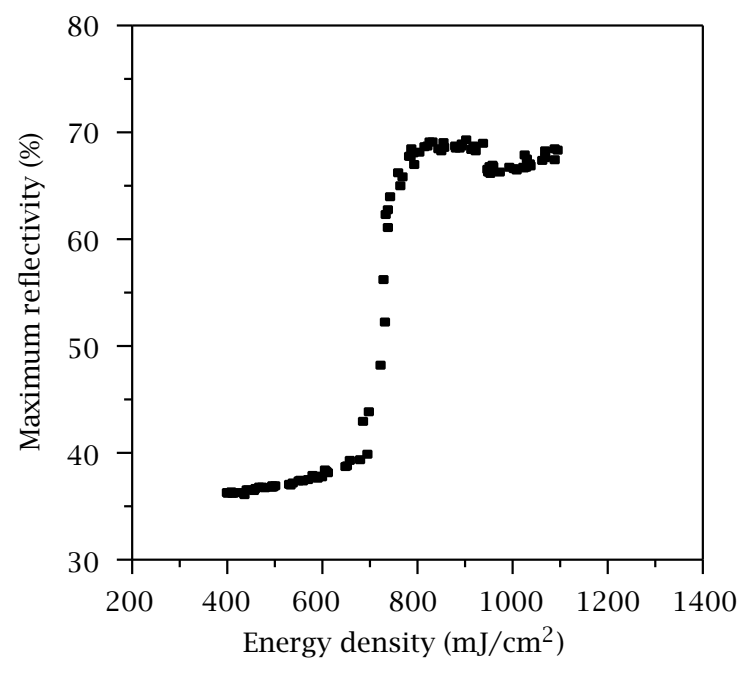

Figure 2. Maximum reflectivity of $\mathrm{He}-\mathrm{Ne}$ laser as a function of energy density for $\mathrm{SiO}_{2}$ sample irradiated with pulsed $\mathrm{XeCl}$ excimer laser. 
Three different regimes can be distinguished.

In regime (A) $\left(E \leq 700 \mathrm{~mJ} / \mathrm{cm}^{2}\right)$ reflectivity slowly increases from the value $35.1 \%$ (reflectivity of the solid $\mathrm{SiO}_{2}$ at temperature $300 \mathrm{~K}$ ) to the value $40 \%$. In Figure 3, these points are represented by TRR spectra 1,2.

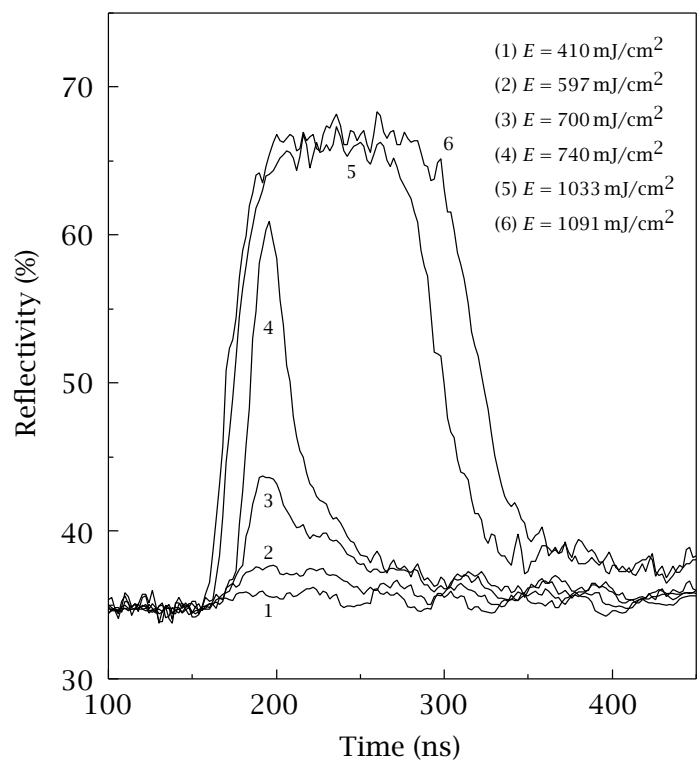

Figure 3. Time-resolved reflectivity spectra of $\mathrm{SiO}_{2}$ Sample at $632.8 \mathrm{~nm}$ during pulsed $\mathrm{XeCl}$ excimer irradiation at different energy densities.

In regime (B) $\left(700 \leq E \leq 800 \mathrm{~mJ} / \mathrm{cm}^{2}\right)$ reflectivity increases rapidly from $40 \%$ to the value $66 \%$ the high reflectivity of the liquid Si . Jellison et al. [15] show that near the melting threshold the High reflectivity phase signal was never less than a certain threshold value and with increasing energy density of the laser pulse the maximum reflectivity continuously increases from its value corresponding to a hot solid to that of a liquid. They proposed that this was due to the inhomogeneous melting of the near-surface layer with a thickness of about $20 \mathrm{~nm}$. Qualitatively similar results from TRR measurements were obtained by Foulon et al. [16] in the case of ArF excimer laser irradiation. They concluded that the reflectivity of liquid Si could be observed only if the molten layer was thicker than certain thickness roughly equal to the absorption length of the probe beam in liquid Si. For thinner molten layers the reflectivity is a function of layer thickness. There is not abrupt change of reflectivity at a particular value of $E$ i.e. reflectivity of a two-layer system (liquid-mono Si) should be considered, maximum reflectivity approaches reflectivity of liquid Si. This part of dependence of maximum reflectivity on energy density is represented in Figure 3 by TRR spectra 3 and 4 .

In regime $(C)\left(E \geq 800 \mathrm{~mJ} / \mathrm{cm}^{2}\right)$ the maximum reflectivity is approximately constant and is equal to $66.5 \%$ this part of dependence is represented by TRR spectra 5,6 Figure 3 . The obtained maximum reflectivity value (66.5\%) is less than maximum reflectivity of liquid $\mathrm{Si}$ at the same energy density this difference could be explained as a follows: intense UV radiation in the surrounding gaseous atmosphere can produce a gasmolecule photolysis that can be effective in improving the reaction rate at the surface

$$
\begin{gathered}
\mathrm{O}_{2}+h v \longrightarrow 2 \mathrm{O}^{*} \\
\mathrm{O}^{*}+\mathrm{SiO} \longrightarrow \mathrm{SiO}_{2}
\end{gathered}
$$

Irradiation of $\mathrm{O}_{2}$ creates active oxygen species such as atomic oxygen, ozone, or ionic oxygen, which react directly with Si surface. The absorption depth at (UV) wavelength is small $(10 \mathrm{~nm})[3,4]$ so that the increase in the thickness of the oxide layer due to change the oxygen content leads to decrease the amount of energy which reach to the surface and consequently the maximum reflectivity decreased. H. Philipp [17] studied the influence of the oxide layers on the determination of the optical properties of Si and reported that the effect of this film is to reduce the reflectance from that of a pure Si. This conclusion is in agreement with our experimental results.

From dependence of maximum reflectivity on energy density in Figure 3, the melting threshold was determined $\left(700 \mathrm{~mJ} / \mathrm{cm}^{2}\right)$. The melting threshold is defined as the intersection of straight lines interpolated through the experimental data in the first part of the curve and the second one.

Maximum reflectivity corresponding to the measured melting threshold $700 \mathrm{~mJ} / \mathrm{cm}^{2}$ is reflectivity of silicon dioxide at the melting temperature and its value is $40 \%$.

The measured time of melting as a function of the incident energy density are shown in Figure 4.

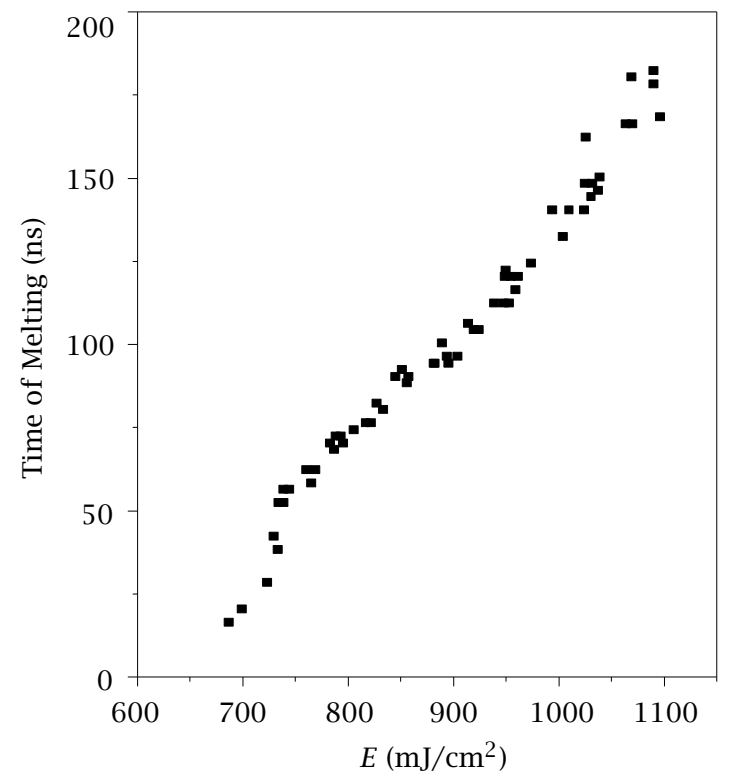

Figure 4. Time of melting as a function of energy density for $\mathrm{SiO}_{2}$ Sample.

These results pointed out the existence of two different melting thresholds. The first one, which is conident with $700 \mathrm{~mJ} / \mathrm{cm}^{2}$, should correspond to the energy density required to nucleate the liquid phase. The sec- 
ond one, evidenced by the slope change at $760 \mathrm{~mJ} / \mathrm{cm}^{2}$ corresponds to the energy density required to induce a homogenous molten layer on top of the surface.

3.3. Phase transformation mechanisms involved in excimer laser crystallization of a-Si : $\mathrm{H}$ thin films. To illustrate the sensitivity of our method we selected the published TRR spectra from reference [7]. Figure 5 illustrates TRR spectra during irradiation of a-Si : H layer with three consecutive excimer laser pulses before calibration.

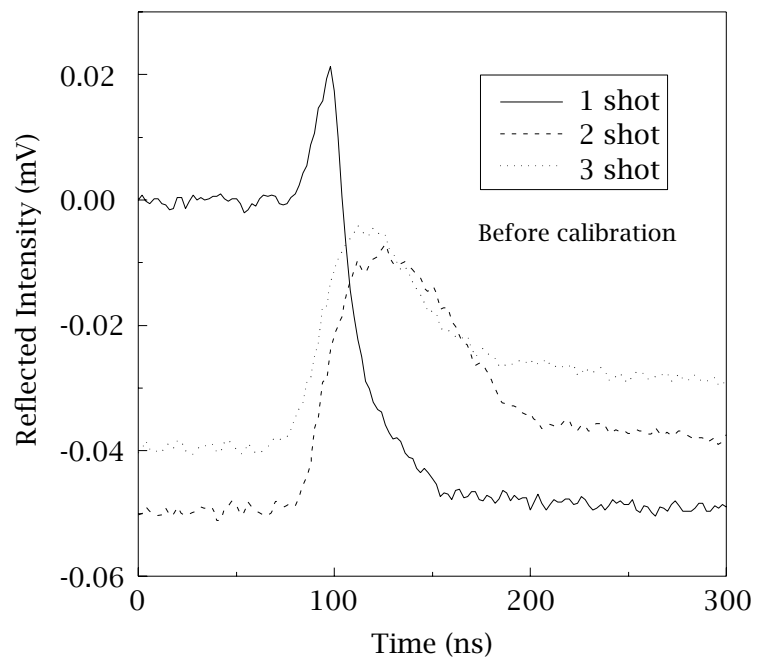

Figure 5. TRR spectra before calibration during irradiation of $\mathrm{a}-\mathrm{Si}: \mathrm{H}$ sample with consecutive excimer laser pulses whose serial number are indicated.

Applying our calibration method the reflectivity column multiplied by factor $F=284$ and the reflectivity of a-Si at room temperature and $632.8 \mathrm{~nm}\left\{R_{\mathrm{S}}=42.5 \%\right.$

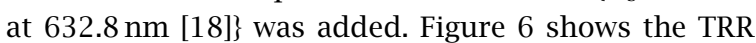
spectra after calibration. The end of TRR spectrum of the first shot is considered as a beginning for the next spectrum and so on. A-Si: $\mathrm{H}$ samples were irradiated with energy density $450 \mathrm{~mJ} / \mathrm{cm}^{2}$, which is high enough to melt the sample surface. For the first pulse the transient reflectivity increased from the reflectivity of a-Si $(42.5 \%$ at $632.8 \mathrm{~nm})$ to the higher reflectivity of liquid phase $48.6 \%$ and after the laser pulse absorbed the reflectivity decreased to $28.5 \%$. This result is evident because the reflectivity of a-Si at melting temperature is $47.6 \pm 0.5$ for the same probe He-Ne laser $(632.8 \mathrm{~nm})$ [18]. The end of the reflectivity of the first pulse was considered as a beginning the second pulse. For the second pulse recrystallization process continue during the absorption of the incident laser pulse the reflectivity increased from $28.5 \%$ to $40 \%$ and after pulse switch off the reflectivity reverse its direction and decreased to $32.2 \%$. For the third pulse the reflectivity increased from $32.2 \%$ to $41.8 \%$ and then decreased to $34.7 \%$. These results indicated that the third pulse was enough to transform the sample from pc-Si phase to crystalline phase. It is well known that the reflectivity of c-Si at melting point $=41.5 \%$ [19] and reflectivity of c-Si at room temperature $=34.7 \%$ [13] for the same probe He-Ne laser.

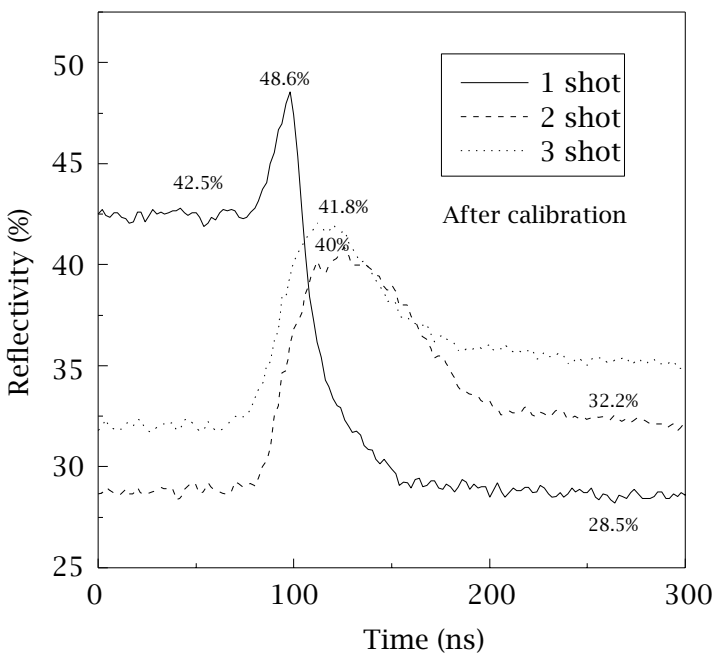

Figure 6. TRR spectra after calibration during irradiation of a-Si : H sample with consecutive excimer laser pulses whose serial number are indicated.

\section{CONCLUSIONS}

Time-Resolved Reflectivity technique was improved via calibration of the reflectivity in TRR spectrum. This method successfully was applied on $\mathrm{SiO}_{2}$ sample, which irradiated in oxygen medium to increase the thickness of oxide layer. Melting threshold energy density was determined to be $700 \mathrm{~mJ} / \mathrm{cm}^{2}$. The maximum reflectivity at melting threshold was determined to be $40 \%$ at He-Ne laser probe beam. Also TRR spectra for a-Si : H samples were calibrated. The obtained results showed that the sensitivity of the method to illustrate the different phase transitions (a-Si, pc-Si and c-Si). This method represents progress in the field of laser annealing semiconductors because:

(1) The analysis of the treated sample with pulsed laser will be done immediately during irradiation, it saves cost and time of analysis.

(2) The problem of the oxidation of the irradiated sample will be finished where it is not need to break ultrahigh vacuum to transfer the sample from this technique to other.

(3) The reflectivity of the semiconductors could be measured at high temperature without using high temperature furnaces, since the molten region is created by laser irradiation.

\section{ACKNOWLEDGEMENTS}

I would like to thank Dr. V. Chab (institute of physics Prague Czech Republic) for his continuous support during the experimental work.

\section{REFERENCES}

[1] O. Krivanek and H. Mazur, Appl. Phys. Lett. 37 (1980), 392.

[2] F. Vega, C. N. Afonso, C. Ortega, and J. Siejika, J. Appl. Phys. 74 (1993), 963.

[3] E. M. Young and W. A. Tiller, Appl. Phys. Lett. 50 (1987), 80. 
[4] C. Fiori, Phys. Rev. Lett. 52 (1984), 2077.

[5] K. M. A. El-Kader, J. Oswald, J. Koèka, and V. Cháb, Appl. Phys. Lett. 64 (1994), 2555.

[6] J. S. Im, H. J. Kim, and M. O. Thompson, Appl. Phys. Lett. 63 (1993), 1969.

[7] K. M. A. El-Kader, I. Ulrych, V. Cháb, J. Oswald, P. Kubat, P. Engst, J. Stuchlik, A. Fejfar, R. Èerný, I. Pelant, and J. Koèka, Thin Solid Film 255 (1995), 302.

[8] K. M. A. El-Kader, O. Borusik, Z. Chvoj, I. Ulrych, J. Oswald, I. Pelant, J. Stuchlik, V. Cháb, J. Koèka, T. Dyer, and J. M. Marshall, Thin Solid Film 276 (1996), 306.

[9] D. H. Auston, J. A. Golovchenko, A. L. Simons, C. M. Surko, and N. C. Venkatesan, Appl. Phys. Lett. 34 (1979), 777.

[10] K. M. A. EL-Kader, Ph.D. thesis, Charles unv. prague, 1995.
[11] K. M. A. El-Kader, Egyptian J. Solids, accepted for publication.

[12] K. M. Shvarev, B. A. Baum, and P. V. Geld, High temperature, 15 (1977), 548.

[13] G. E. Jellison, Jr. and F. A. Modine, J. Appl. Phys. 76 (1994), 3758.

[14] N. Terada, T. Haga, N. Miyata, and K. Moriki, Phys. Rev. B 46 (1992), 2312.

[15] G. E Jellison, Jr., D. H. Lowndes, D. N. Mashburn, and R. F. Wood, Phys. Rev. B 34 (1986), 2407.

[16] F. Foulon, E. Fogarassy, A. Slaoui, C. Fuchs, S. Unamuno, and P. Siffert, Appl. Phys. A. 45 (1988), 361.

[17] H. R. Philipp, J. Appl. Phys. 43 (1972), 2835.

[18] D. H. Lowndes, S. J. Pennycook, G. J. Jellison, Jr., S. P. Withrow, and D. N. Mashburn, J. Mater. REs. 2 (1987), 648.

[19] G. E. Jellison, Jr. and H. H. Burke, J. Appl. Phys. 60 (1986), 841. 


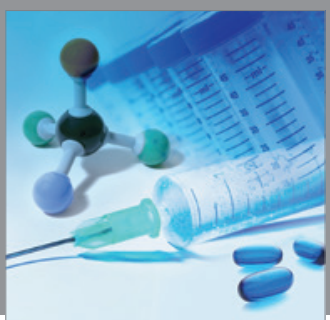

International Journal of

Medicinal Chemistry

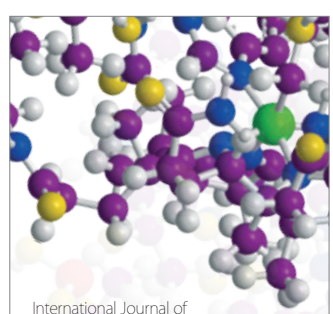

Carbohydrate Chemistry

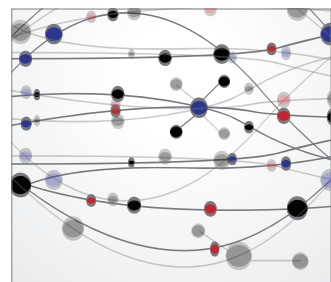

The Scientific World Journal
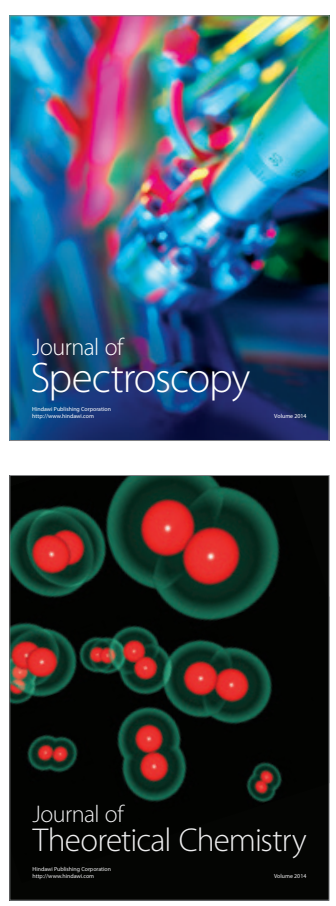
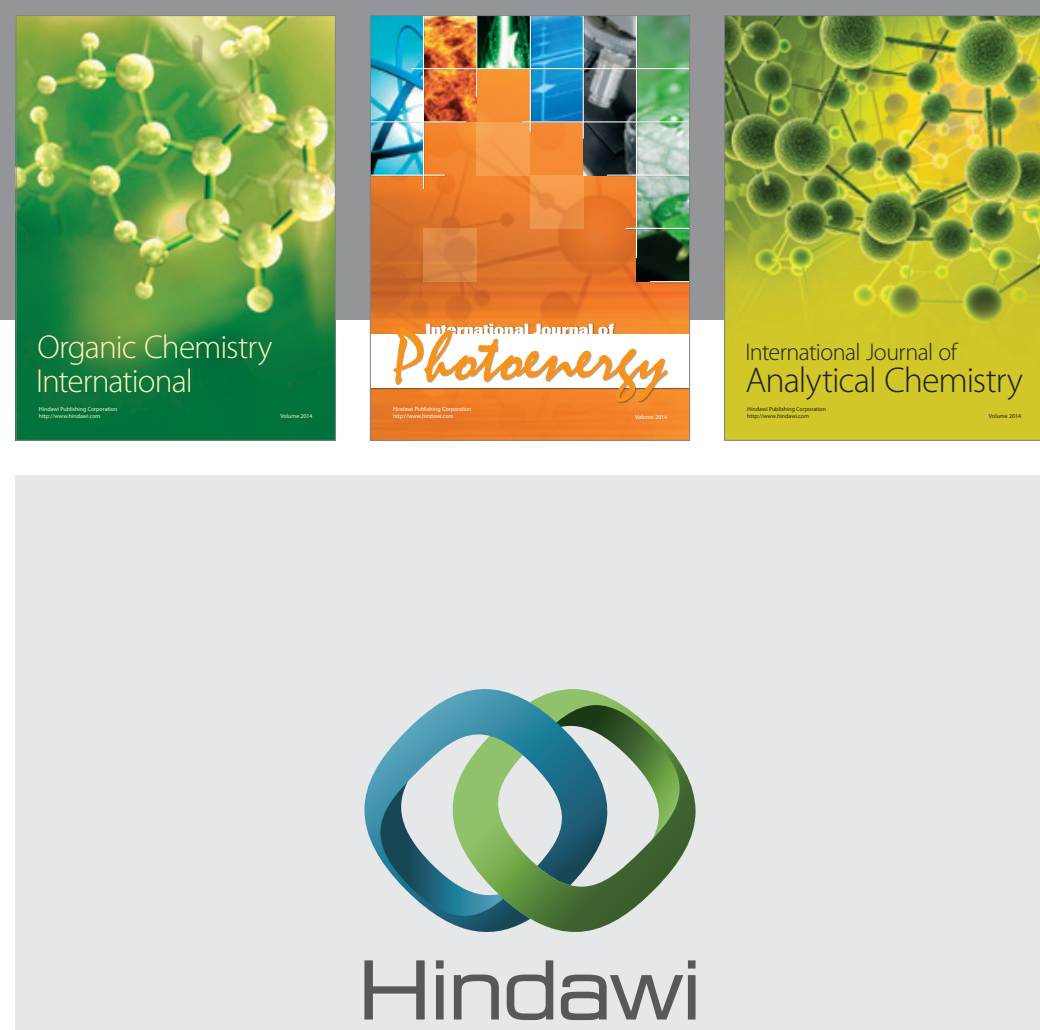

Submit your manuscripts at

http://www.hindawi.com
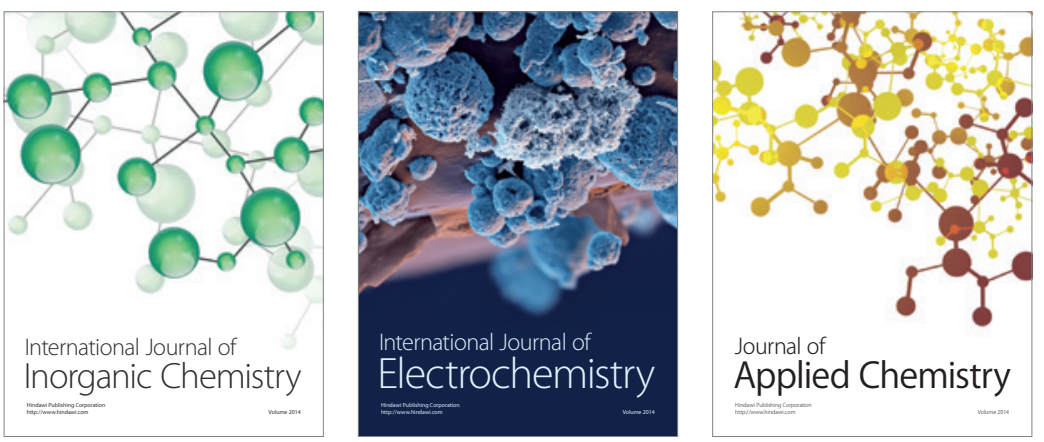

Journal of

Applied Chemistry
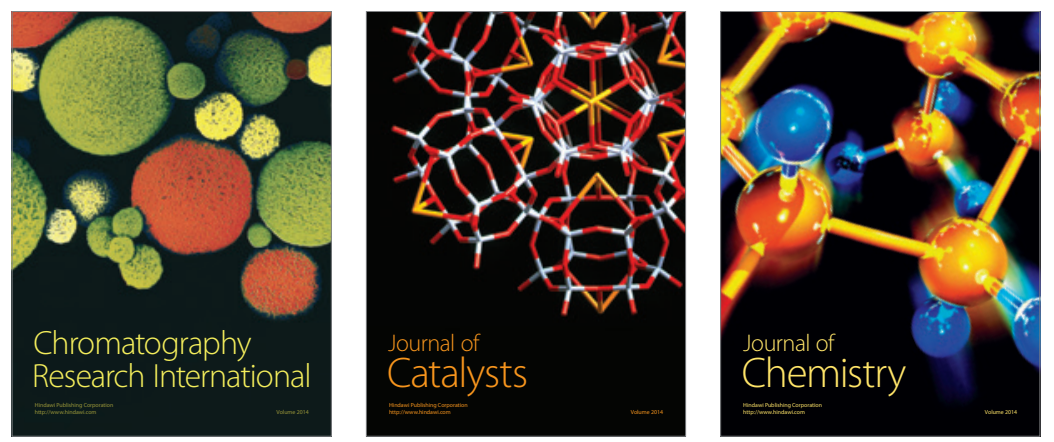
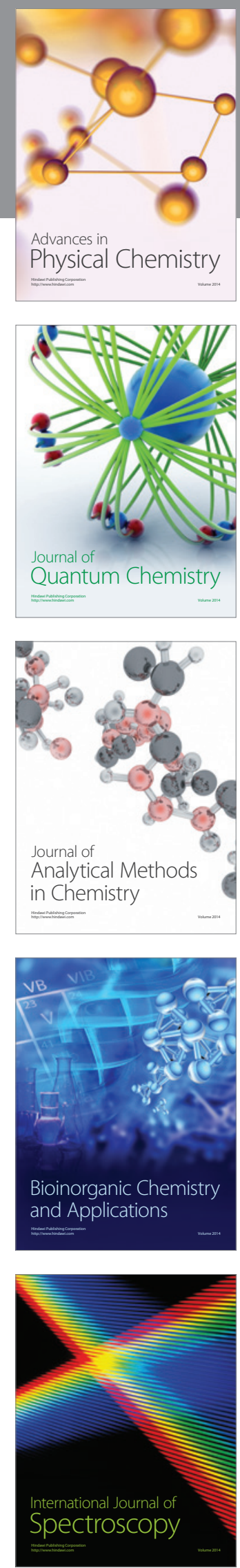